\title{
LEUCEMIA MONOCITICA AGUDA EN EL NIÑO
}

\author{
Por los Dres. JOSE LEGARRETA. CARLOS RIOS y RAUL ERAZO \\ Hospital L. Calvo Mackenna. Cátedra de Pediatria del Prof. A. Ariztía.
}

La leucemia monocítica aguoda constituye una forma rara, si bien no excepcional, de leucemia en la infancia.

Hasta el año 1950, en el Hospital Luis Calvo Mackenna no se registra ninguna observación de este tipo, pero en el curso del año 1951 hemos tenido ocasión de observar dos casos en nin̄os hospitalizados en el Servicio de Medicina Interna.

Han sido los trabajos de Reshap y Schilling-Torgau en 1913, los primeros en diferenciar este tjpo de leucemia, basados en estudios morfológicos. Clough hace una revisión de la bibliografía publicada hasta 1932 con un total de 23 observaciones en adultos. Desde esa época se han descrito numerosos casos, tanto en adultos como en niños, en la literatura mundial.

Existe, sin embargo, cierta confusión en lo que a terminología se refiere; esto se debe al hecho de no existir uniformidad de criterio respecto al origen de los monocitos. Así, algunos autores han usado los términos de reticulosis o retículoendoteliosis como sinónimos de leucemia monocítica, al asignarle al monocito su origen en el S. R. E. Igualmente las llamadas leucemias hemohistioblásticas han sido consideradas por otros, leucemias monocíticas.

A su vez, Naegeli, Piney, Anagnosi, etc. consideran al monocito derivado del mieloblasto y no aceptan la leucemia monocítica como una entidad distinta, sino como una fase transitoria de la leucemia mielógena.

La característica hematológica de este cuadro es la presencia en la sangre circulante, de gran número de células de la serie monocítica, constituyendo el $60 \%$ o más del total de los leucocitos.

Downey resume los conceptos anteriores, distinguiendo dos variedades de leucemia monocítica: el tipo Schilling, en la que el monocito deriva del S. R. E. y el tipo Naegeli, siendo aquí el monocito de origen mieloblástico. Esta distinción es sólo hematológica, no existiendo diferencias significativas con respecto al cuadro clínico.

En la variedad Naegeli, la sangre periférica tiene gran cantidad de monocitos en diversas etapas de desarrollo, junto a mieloblastos y mielocitos jóvenes. En el tipo Schilling nay predominio de monocitos y pueden encontrarse histiocitos grandes, pero no se observan mieloblastos en la sangre periférica.

$\mathrm{La}$ incidencia con respecto a la edad varía entre los 5 y 70 años. Grenet-Levent e Isaac-Georges citan dos casos en niños de $2 \frac{1}{2}$ y 3 años, respectivamente. En nuestro pais, Costa, en un completo estudio sobre leucemias en la infancia, refiere 3 casos.

La mayor parte de las leucemias monociticas tienen un curso agudo o subagudo, en el $11 \%$ es crónica, con evolución que puede llegar a los 6 años.

Entre los síntomas clínicos más frecuentes de las formas agudas, se citan los siguientes: lesiones de la boca (gingivitis, anginas, úlceras y necrosis); púrpura y hemorragia de las mucosas. La hiperplasia linfática es moderada y puede faltar, es más notoria en el cuello. La esplenomegalia y hepatomegalia no son intensas. 
Como hallazgos hematológicos se señalan leucocitosis que varian entre 25,000 y 100,000 elementos, con predominio de la serie monocítica (60 a $90 \%$ de las células), pero también puede haber leucopenia. Se encuentra, además, anemia progresiva $\mathrm{y}$ trombopenia, esta última explica en parte las manifestaciones purpúricas y hemorrágicas.

En el estudio histopatológico llama la atención las infiltraciones extensas de los órganos, especialmente bazo, hígado y ganglios, por células monocíticas aná-
En el Servicio el nfño continúa febril, aparecen úlceras peridentarias cubtertas de falsas membranas $y$ un flegmón en la parte izquierda del maxilar inferior. Hígado y bazo a un centimetro del reborde. Sedimentación: $168 \mathrm{~mm}$ en la hora.

Hemograma: 2.180,000 glóbulos rojos con 76,500 glóbulos blarcos; hemoglobina, $4 \mathrm{~g}$. Fin la formula hay 11 mieloblastos y 2 promonocitos.

Se trata con transfusiones y penicilina, consiguiéndose la curación del flegmón de la boca y desaparición de la fiebre.

Posteriormente tlene adenitis $y$ periadenitis submaxilar, eplstaxis y brote purpúrico de carácter progresivo; además, aparecen ele-

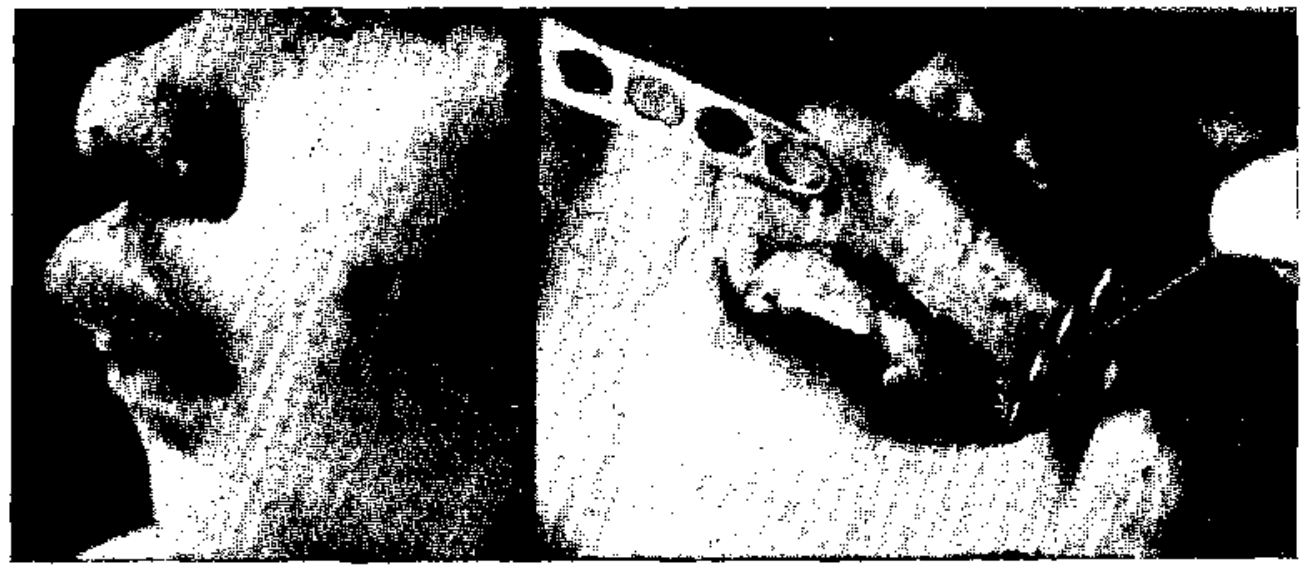

Obs. 51-1259.-Lesiones úlceronecróticas de la boca.

logas a las de la sangre. Clough anota dos hechos fundamentales en el cuadro anátomopatológico: 1) aumento e hiperplasia de las células monocíticas en el tejido conjuntivo, especialmente alrededor de los vasos sanguíneos, y 2) hiperplasia de células de tipo endotelial en bazo, hígado, ganglios y aun en médula ósea.

\section{Casuística.}

CASO $N^{9} 1$ - Observación 51-1250.

Niño de 9 años de edad que ingresó al Servicio en marzo de 1951.

Sn enfermedad actual se habría intelado un mes antes con anorexia, istenia, adinamla, flebre e infarto ganglionar del cuello; además, tuvo epistaxis. En marzo de 1951 consulta en policlínica, encontrándose un niño con mal estado general y nutritivo, febril, decaido y pálido, con edema del labio superior, aliento fétido, caries dentarias y glngivitis necrótica. Micropolladenia. mentos pápulonecróticos acneiformes en la cara, especialmente en labio superior y nariz.

En las últimas semanas de hospitalización empeora el estado general, tlene fiebre alta, abscesos múltiples, el higado y el bazo se palpan a $5 \mathrm{~cm}$ del reborde. BS retirado del Servlclo por sus famillares y días después fallece en su casa (mayo de 1951).

Los hemogramas posteriores confirman el diagnóstico de leucemia monocitica; la cífra de leucocitos varía entre 35,000 y 100,000 elementos por $\mathrm{mm}^{3}$. La fórmula se caracteriza por el gran aumento de los monocitos (22 al $44 \%)$ y presencia constante de promonocitos que fluctúan entre el 34 y $51 \%$. En el último hemograma la cifra total de elementos de la serie monocítica alcanzó al $89 \mathrm{r}$. Las plaquetas estaban considerablemente disminuídas y al final, casi abolidas.

En los mielogramas se observó también gran predominio de células de la serie monocítica $64 \%$ de promonocitos y $17 \%$ de monocitos). En una radiografía de huesos largos se encuentran infiltraclones epjflsiarias de tipo leucémico. 


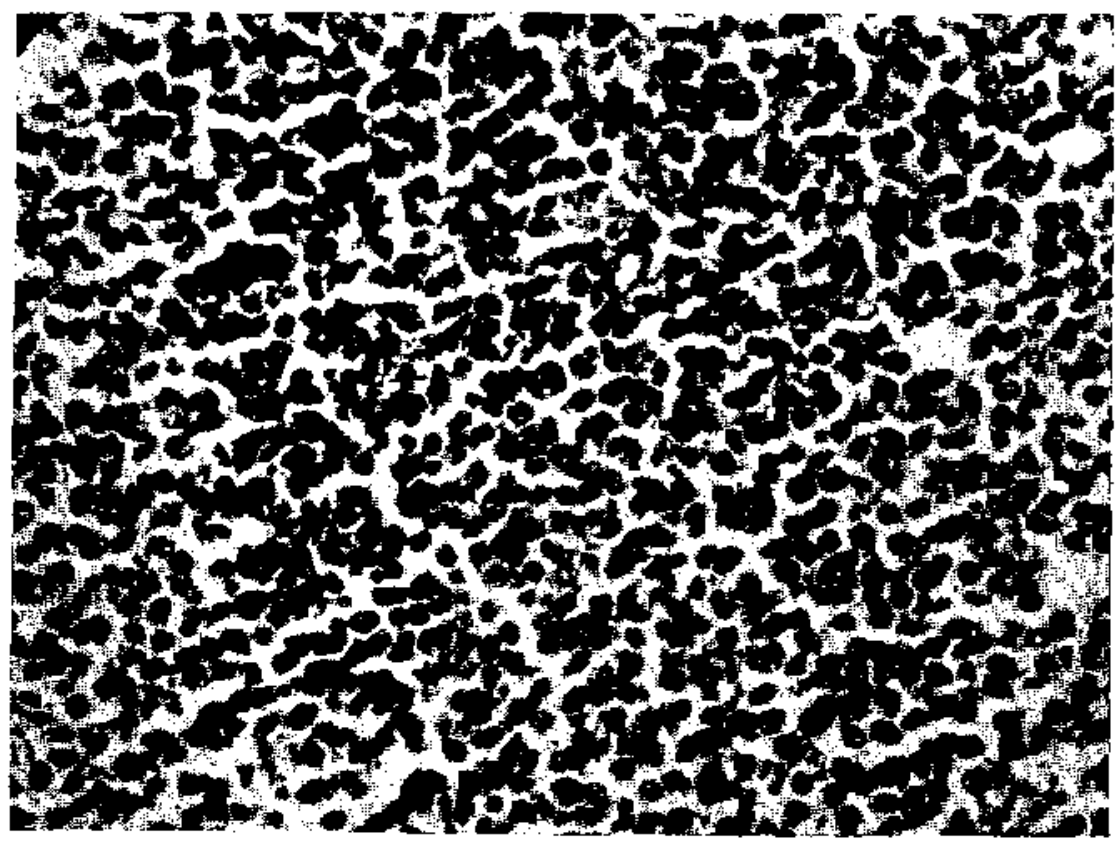

Cbs. 51-4789.--Infiltraçón leưémíca del bizo.

CASO $\mathrm{N}^{4} 2$ - Observación 51-4789.

Nln̄o de 10 años de edad; ingresó al SerFlelo en septiembre de i.55i por presentar desde una semana antes elementos purpúri- cos petequiales en las extremidades superiore3 y tronco; pcsteriormente tiene fiebra, decaimiento, anorexia y epistaxis.

Tres semanas antes a su hospitalización fué vacunado con BCG.

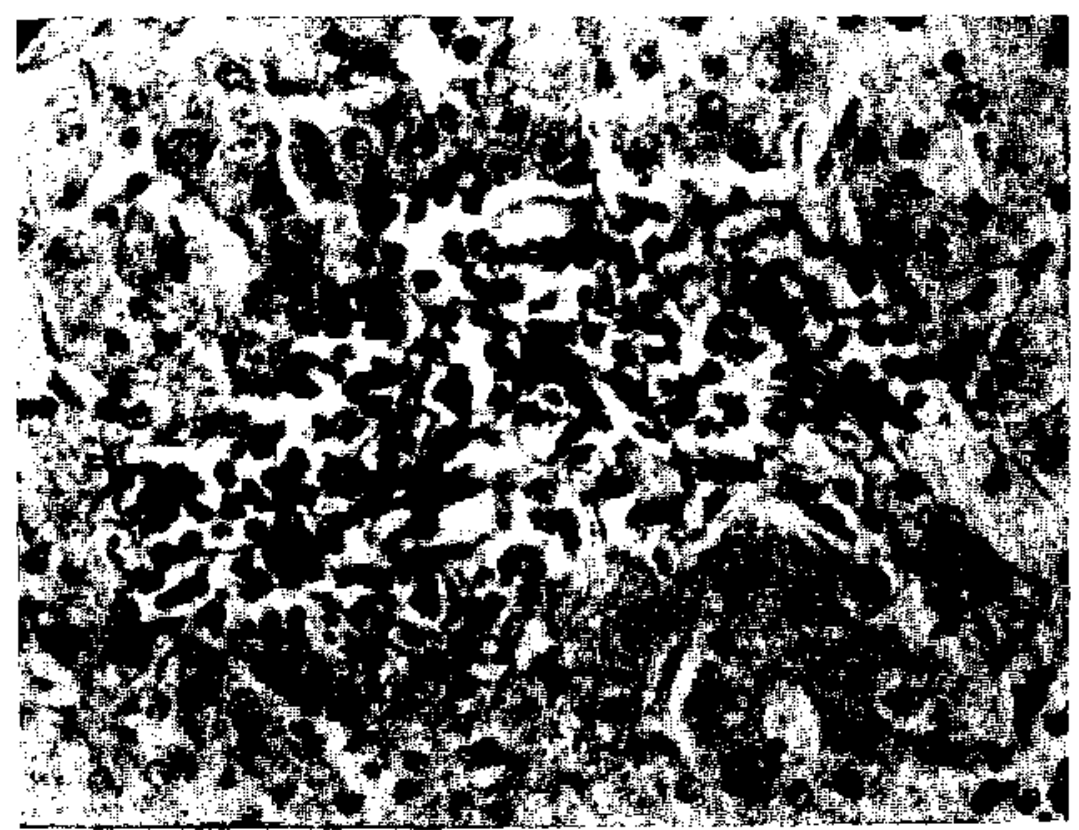

Obs. 51-4789. -Infiltración leucémica del hígado. 
Antecedentes sin importancia. En el exa la etiología del púrpura. La trombopemen se encuentra un nin̄o con regular estado nutritivo, decaido. Piel con elementos purpúricos diseminados, más abundantes $€ n$ el hombro izquierdo. Micropoliadenia. Edema palpebral discreto. Resto nada de especial.

Hemograma: Anemia de 3 millones de glóbulos rojos; leucocitosis de 20,000 glóbulos blancos; 1,400 plaquetas. En la fórmula se cuentan $7 \overline{5}$ promonocitos con 22 linfocitos $y$ 3 segmentados.

Evoluciona con fiebre alta $y$ a las 2 semanas de hospitalización aparecen dolores intensos en Ia pierna izquierda y pasteriormente en el musio derecho, de tipo osteóscopo, que obligan al uso de analgésicos (Sedol, Cilagmine, etc.). El mielograma confirma el diagnóstico de leucemla monocitica.

Al mes de hospitalízación sube la fiebre a 40" y aparecen lesiones úlceno-necrótlcas de Ia boca, especialmente en encias $y$ faringe; tiene epistaxis y nuevo brote purpúrico; además, se queja de dolores intensos localizados ahora en el brazo izquierdo.

Se trata con transfusiones y penicilina para evitar la infección secundaria de sus lesiones bucales. Las plaquetas están casi abolidas y hay leucopenia progresiva que llega a 2,000 glóbulos blancos.

El 19 de norlembre tiene un ataque convulsivó generalizado con pérdida del conocimiento, con equimosis del ojo derecho y hemorragias peripapilares. Cuatro días después se repite el ataque convulsivo, el niño cae en coma $y$ fallece.

Fn la necropsia se encuentran hemorraglas de serosas $\mathrm{y}$ mucosas, gran hemorragta encefálica del centro oval lzquierdo con inundación ventricular y microhemorragias encefálicas múltiples. Médula ósea achocolatada y compacta con estructura perdida por invastón de elementos leucémicos. Infiltración leucémica del rifón e hígado. Hiperplasla. linfática de ganglios $y$ bazo.

\section{Comentario clínico.}

En ambos casos llama la atención la presencia de fenómenos hemorrágicos diversos y de intensidad progresiva; las epistaxis ya habían hecho su aparición antes de la hospitalización de los niños. En el caso II los elementos purpúricos eran más confluentes alrededor del sitio de vacunación con BCG, lo que hizo pensar que hubiera cierta relación de causa a efecto entre ambas. Posteriormente, al comprobarse elementos leucémicos en el hemograma, se aclaró

nia marcada que presentaba este niño ha favorecido indudablemente la aparición de nuevas sufusiones sanguíneas, que culminaron con la hemorragia cerebral, causa determinante de la muerte.

Las lesiones úlceronecróticas aparecen en ambos casos alrededor de un mes. después de la iniciación de las molestias y adquirieron mayor intensidad en el primer niño, acompañándose de fenómenos inflamatorios secundarios intensos, extendiéndose, además, a la piel de la cara. Estas lesiones han sido consideradas como específicas de la leucemia monocítica aguda, por algunos autores (Forkner, Merger, etc.) y suelen ser la primera manifestación clinica de la enfermedad. Para otros (Hubler, Netherton, Wintrobe, etc.), tales trastornos son frecuentes (hasta el $50 \%$ de los casos), pero no tienen caracteres de especificidad.

Respecto a los dolores de las extremidades que aquejaron al segundo niño, no se acompañaron de signos inflamatorios, el estudio radiológico fué negativo y se interpretaron como producidos por el aumento de la presión intramedular. provocados por los focos leucémicos o hemorragias intramedulares.

Las lesiones óseas en el curso de las leucemias son más frecuentes en el niño que en el adulto por la mayor actividad hematopoyética (Hasbun, Eberhard). Pueden simular cuadros de reumatismo, artritis $y$ aun osteomielitis. Los infiltrados leucémicos producen osteolisis, desprendimientos del periosteo, cuya irritación es a veces la causa del dolor. En el primer caso obturimos la comprobación radiológica de la infìltración leucémica en los huesos largos.

\section{Comentario hematológico.}

En el primer caso la impresión al esdiar el hemograma fué de una leucemia mieloide crónica, por la gran reacción mielocítica, pero posteriormente la afección derivó francamente hacia la leucemia monocítica (promonocítica tipo Naegeli). Se observa en las preparacio- 
nes abundancia de monocitos maduros y transición entre células maduras e inmaduras, lo que no se observa en las leucemias mieloblásticas agudas. El estudio del mielograma reveló gran proporción de células inmaduras (paramieloblasto de Naegeli) de tipo promonocítico. Las células típicas de esta afección son: monoblastos, promonocitos $\mathrm{y}$ monocitos.

Este caso probaría la teoría de Naegeli y Rosenthal, que dice que la leucemia monocítica seria una fase monocítica de la leucemia mieloide, por el aspecto que presentó al ingreso.

En el segundo caso se encuentra en la sangre al primer examen una anemia de $2.890,000$ con $8.9 \mathrm{~g}$ de $\mathrm{Hb}$. y una leucocitosis de 20,300 con gran predominio de células inmaduras de núcleo arriñonado o polimorfo, con red cromatínica poco densa, protoplasma azul pálido con granulaciones azurófilas y reaeción de peroxidasas a veces escasamente positiva (promonocitos). En la médula ósea se encuentra gran proporción de estas mismas células con aspecto más primitivo. Posteriormente presenta el enfermo una leucopenia acentuada con linfocitosis relativa y disminución de la célula patológica, hasta el momento de su fallecimiento.

\section{Resumen.}

Se hace una revisión bibliográfica de las leucemias monociticas agudas en el nin̄o.

Se presentan dos observaciones de esta enfermedad en niños de 9 y 10 años de edad, destacando las lesiones úlceronecróticas de la boca, los procesos hemorrágicos múltiples y las manifestaciones óseas.

\section{Summary.}

A bibliografic review of the acute monocitic leuckemias in children is undertaken.

Two observations of this illness in children of 9 and 10 years of age are reported, stressing the ulceronecrotic lesions of the mouth, the multiple hemorragic processes and the bone manifestations.

\section{Bibliografia.}

BARRIGA, GOYENECHE y DUCACH, - Rev. Méd. de Chile 67: 131-33, 1939.

BRENNEMANN. - Practice of Pediatrics. W. F. Pritor. Co. Inc. Vol, III. 1948.

CAMPBELT, A. C. P. - J. Path, and Bact. 42: 617, 1936.

CLOUGH, P. W. - Bull. John Hopkins Hos. 51: $148,1932$.

COSTA, A. - Archivos Hospital R. del Río. 1947.

DAMASHEK, - Folia Hemat. 49: 64, 1933.

DOAN, C. A. and WISEMAN, B. K. - Ann. Int. Med. 8: 381, 1934.

DOWNEY, H. - Handbook of Hematology. J. B. Hoeber Ine. N. York, 1938.

FOORD, A. G.; PARSONS, L. - J. A. M. A. 101: 1859, 1933.

FORJENER, C. E. - Arch. Int. Med. 53: 1, 1934.

HASBUN, J. y EBERHARD, R. - Rev. Chilena de Ped. 21: '289-97, 1950.

HUBLER, W. R and NETHERTON, E. W. J. A. M. A. 157: 456, 1947.

ISAACS, R. - Arch. Path. 9: 1298, 1930.

KIRShraUAiv, J. and PREUSS, F. - Int. Med. Digest. 43: $222,1943$.

IAUCKINER and HEBBERT. - J. A. M. A. $136: 723,1948$.

LEVINE, V. - Folia Haemat 52: 305, 1934.

NAEGELI. - Tratado de Hematología. Ed. Labor, 1936.

NELSON. - New Loose Ineaf Medicine. Th. Nelson and Sons. Vol. IV.

OSGOOD, E. E. - Arch. Int. Med, 59: 931, 1937.

POWFIL, W. N. - Int. Med. Digest. 38: 33, 1941.

RAPPAPORT, A, E. and KEEGEL, V. H. Blood. Vol. II: 332, 1947.

RHINEHART, J. F. - Arch. Path. 13: 889, 1932.

RIIS, J. - Quarterly Review of Pediatrtes 2: $602-3,1947$.

SCHILLINGG. - El cuadro hemático y su Interpretación clínica. Ed. Labor. 1936.

WAINWRIGHT, $C, W$. and DUF', G. L. Bull. John Hopkins Hospital. 58: 267, 1936.

WINTROBE, M. M. and MITCHELL, D. M. Quart. J. Med. 9: 67, 1940.

WINTROBE, M. - Hematologia Olinica. Ed. Interamericana s. A. Méxtco. 1948. 2* Edíción.

WOLKINS, C. H. and HOLL, B. E. - J. Clin. Path. 10: 387, 1940. 